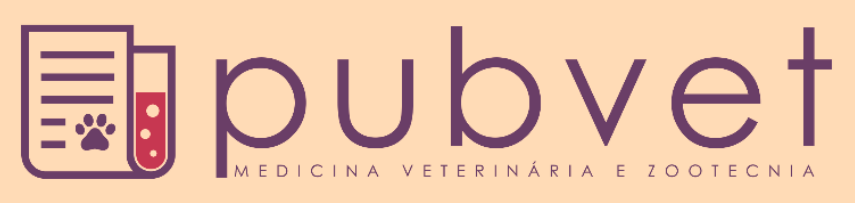

https://doi.org/10.31533/pubvet.v14n2a514.1-7

\title{
Correção cirúrgica de hérnia inguinal com histerocele e maceração fetal: relato de caso
}

\author{
Lucas Matheus Possamai ${ }^{*} \bullet$, Maria Cecilia de Lima Rorig ${ }^{\bullet}$, Renato Herdina \\ Erdmann $^{2} \theta$, Denner Francisco Tomadon Fiorin ${ }^{3}{ }^{\ominus}$, Caio Henrique Ferreira ${ }^{4} \bullet$ \\ ${ }^{I}$ Discente do curso de Medicina Veterinária da Pontifícia Universidade Católica do Paraná. Toledo - PR Brasil. \\ ${ }^{2}$ Médico (a) veterinário(a) docente do curso de Medicina Veterinária da Pontifícia Universidade Católica do Paraná. Toledo - PR Brasil. \\ ${ }^{3}$ Médico veterinário aprimorando no departamento de clínica médica e cirúrgica de pequenos animais na clínica veterinária universitária \\ (clivet) da Pontifícia Universidade Católica do Paraná. Toledo - PR Brasil. \\ ${ }^{4}$ Discente do curso de Medicina Veterinária da Pontifícia Universidade Católica do Paraná. Toledo - PR Brasil. \\ *Autor para correspondência: matheus.possamai@pucpr.edu.br
}

\begin{abstract}
Resumo. Hérnias inguinais formam-se quando órgãos se deslocam através do anel inguinal, ocorrendo com maior frequência em cadelas não castradas de meia idade. A caracterização de histerocele ocorre quando o conteúdo presente nesta hérnia é o útero. O objetivo foi relatar o atendimento de uma cadela adulta com diagnóstico de hérnia inguinal cujo conteúdo era o útero com fetos macerados. O animal chegou a Clínica Veterinária da PUCPR apresentando aumento de volume na região abdominal e sangramento vulvar. o exame físico apresentou baixo escore corporal, desidratação, taquicardia, com conteúdo compatível com fragmentos ósseos irredutíveis a manipulação da hérnia inguinal, o diagnóstico sucedeu-se por meio de radiografia abdominal a qual revelou presença de duas estruturas fetais com calcificações ósseas desestruturadas. A paciente foi internada visando estabilização do quadro clínico e posteriormente foi submetida a herniorrafia e ovariosalpingohisterectomia. Após os procedimentos cirúrgicos estabeleceu-se internamento por mais 24 horas, após esse período institui-se a alta médica. $\mathrm{O}$ retorno foi marcado para 10 dias após alta médica onde a ferida cirúrgica apresentou aspectos de cicatrização sem evidências de secreções. Este trabalho demonstra a importância do diagnóstico e os mecanismos necessários para a estabilização do paciente e a correção cirúrgica para alcançar o sucesso terapêutico.
\end{abstract}

Palavras chave: histerocele, herniorrafia, ovariosalpingohisterectomia

\section{Surgical correction of inguinal hernia with hysterocele and fetal maceration: case report}

\begin{abstract}
Inguinal hernias form when organs move through the inguinal ring, occurring most often in middle-aged non-castred animals. The hysterocele occurs when the content present in this hernia is the uterus. The objective of this study was to report the care of an adult female dog diagnosed whit inguinal hernia whose content was the uterus with macerated fetuses inside. The animal arrived at the PUCPR Veterinary Clinic with increased volume in the abdominal region and vulvar bleeding. Physical examination showed low body score, dehydration, tachycardia, with content compatible with irreducible bone fragments and manipulation of the inguinal hernia. The diagnosis was made by amdominal radiography, which revealed the presence of two fetal structures with unstructured bone calcifications. The patient was hospitalized to stabilize the clinical condition and later underwent herniorrhaphy and ovariosalpongohisterectomy. After the surgical procedures, the patient was admitted for another 24 hours, with the medical
\end{abstract}


discharged instituted after that period. The return was scheduled for 10 days after medical discharge where the surgical wound presented healing aspects without evidence of secretions. This paper demonstrates the importance of diagnosis and the mechanisms required for patient stabilization and surgical correction to achieve therapeutic success.

Keywords: hysterocele, herniorrhaphy, ovariosalpingohisterectomy

\section{Corrección quirúrgica de la hernia inguinal con histerocele y maceración fetal: reporte de un caso}

Resumen. Las hernias inguinales se forman cuando los órganos se mueven a través del anillo inguinal, ocurriendo con mayor frecuencia en perras no castradas de mediana edad. La caracterización del histerocele ocurre cuando el contenido presente en esta hernia es el útero. El objetivo fue relatar el atendimiento de una perra adulta diagnosticada con hernia inguinal cuyo contenido era el útero con fetos macerados. El animal llegó a la Clínica Veterinaria PUCPR con aumento de volumen en la región abdominal y sangrado vulvar. El examen físico mostró bajo puntaje corporal, deshidratación, taquicardia, con contenido compatible con fragmentos óseos irreductibles y manipulación de la hernia inguinal. El diagnóstico se realizó mediante radiografía abdominal, que reveló la presencia de dos estructuras fetales con calcificaciones óseas no estructuradas. La paciente fue hospitalizada para estabilizar la condición clínica y luego se sometió a herniorrafia y ovariosalpingohisterectomia. Después de los procedimientos quirúrgicos, el paciente ingresó otras 24 horas, luego de lo cual la se instituyó el alta médica. El regreso se programó para 10 dias después de la alta médica donde la herida quirúrgica presentaba aspectos curativos sin evidencia de secreciones. Este trabajo demuestra la importancia del dignóstico y los mecanismos necesarios para la estabilización del paciente y la corrección quirúrgica para lograr el éxito terapêutico.

Palabras clave: histerocele, herniorrafia, ovariosalpingohisterectomia

\section{Introdução}

A hérnia inguinal é formada quando ocorre protusão de órgãos pelo anel inguinal (Bellenger, 1980; Costa Neto et al., 2006; Ramírez et al., 2015). Elas podem ser falsas ou verdadeiras, sendo que as verdadeiras manifestam o saco e o anel herniário composto por peritônio parietal, o qual circunda o conteúdo. As falsas por sua vez, apresentam o saco herniário formado por pele, subcutâneo e fáscia (Penaforte Júnior et al., 2017). As hérnias inguinais são classificadas como diretas ou indiretas, sendo que as indiretas são aquelas que penetram a cavidade do processo vaginal e as diretas são incomuns e ocorrem quando as vísceras adentram e repousam no subcutâneo através do anel inguinal. Estas apresentam maior volume e por conta disso dificilmente estrangulam (Bellenger, 1980; Cousty et al., 2010; Daleck et al., 1992; Slatter, 2007).

A histerocele inguinal ocorre quando o conteúdo envolvido na hérnia é o últero, a qual apresenta evidente aumento de tamanho com o decorrer da gestação em um ou ambos cornos uterinos contidos no saco herniário e isso causa distocia (Fossum, 2014; Sturion et al., 2013). Fossum (2014) afirma que o útero irredutível, não gravídico dentro do saco herniário dificilmente resultará em complicações, diferentemente de um útero gravídico ou com patologia associada. Este por sua vez será capaz de causar alterações sistêmicas como toxemia e ruptura uterina.

A ocorrência de um processo séptico no útero durante a gestação gera a maceração fetal, resultando em esqueletização devido à liquefação e amolecimento do feto (Toniollo \& Vicente, 2003). Uma das principais causas da maceração fetal é o uso indevido de contraceptivos (Loretti et al., 2004). Booth \& McDonaldo (1992) afirma que, o uso de contraceptivos faz com que os níveis de progesterona se mantenham altos, ocasionando relaxamento da cérvix e não expulsão do feto.

Este relato tem por objetivo apresentar um caso de correção cirúrgica de hérnia inguinal com histerocele e maceração fetal em uma cadela, vista a importância e escassez de relatos anteriores. 


\section{Relato de caso}

Foi atendida na Clínica Veterinária Universitária (Clivet) da Pontifícia Universidade Católica do Paraná, uma cadela sem raça definida, adulta, pesando 10,7 kg. A queixa principal apresentada pelo tutor era um aumento de volume em região abdominal e sangramento vulvar com maior intensidade nos últimos sete dias. Ainda segundo o tutor a paciente apresentava normorexia e normodipsia. Havia sido administrado contraceptivo nos últimos dois anos.

Ao exame físico constatou-se baixo escore corporal, mucosas hipocoradas, desidratação moderada de $8 \%$, frequência cardíaca de 160 batimentos por minuto (bpm), tempo de preenchimento capilar (TPC) de dois segundos, temperatura retal de $39^{\circ} \mathrm{C}$ e linfonodos submandibulares e pré-escapulares alterados bilateralmente. Durante a palpação abdominal foi possível observar grande aumento de volume em região inguinal esquerda, irredutível e à manipulação da região percebeu-se conteúdo compatível com pequenos fragmentos ósseos além de presença de secreção vaginal sanguinolenta, suspeitando-se assim de maceração fetal.

Como exames complementares solicitaram-se hemograma e bioquímica sérica, os quais não apresentaram alterações significativas. Além disso, foi realizada radiografia abdominal nas posições ventro dorsal (VD) e lateral, nas quais foi observado presença de saco herniário em região inguinal esquerda, com visualização de alças intestinais e duas estruturas fetais com calcificação óssea desestruturadas, sugerindo mumificação ou maceração fetal (Figura 1).

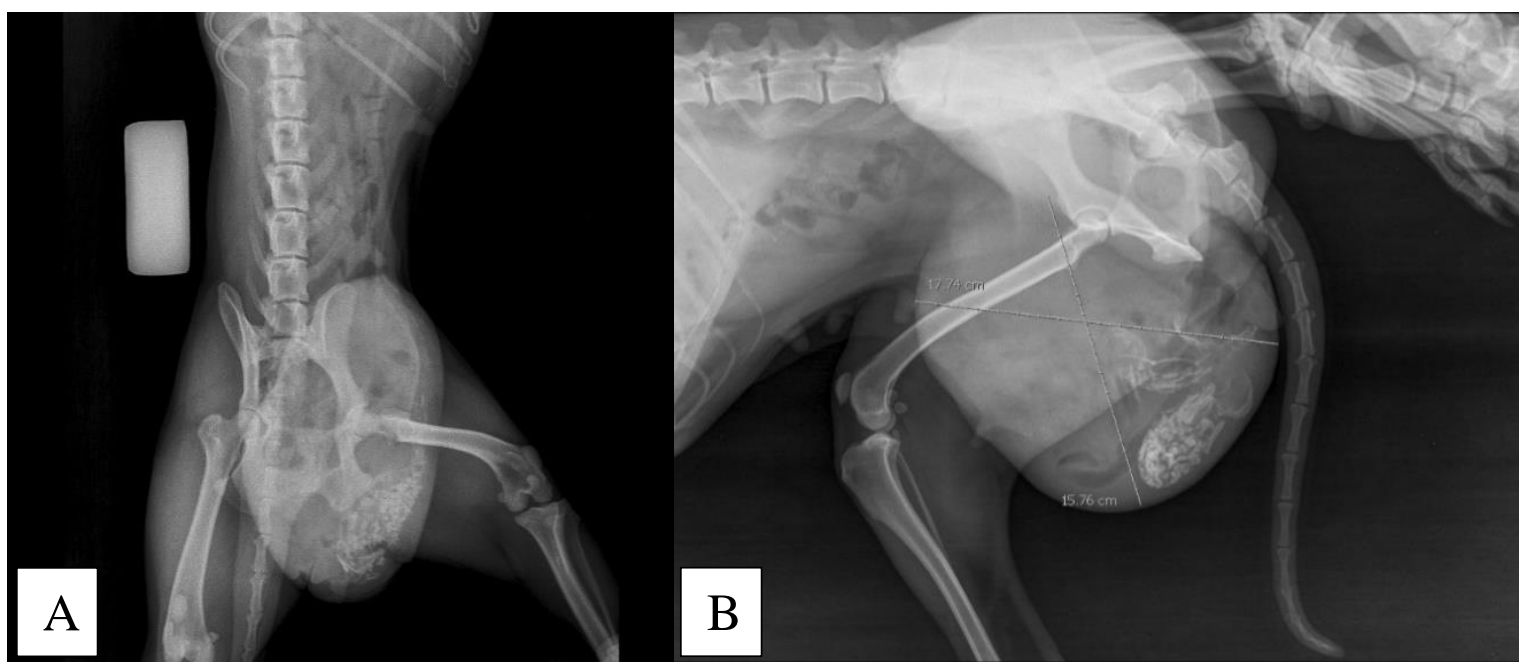

Figura 1. Radiografia abdominal com visualizações de alças intestinais e duas estruturas fetais com calcificação óssea desestruturadas no saco herniário. A. posicionamento ventro dorsal (VD). B. posicionamento lateral.

Diante da gravidade do quadro e da condição clínica da paciente, a conduta inicial foi o internamento para estabilização e posteriormente procedimento cirúrgico de herniorrafia inguinal associado a ováriosalpingohisterectomia (OSH). No internamento o animal recebeu fluidoterapia com solução de ringer com lactato, tramadol $4 \mathrm{mg} / \mathrm{kg} / \mathrm{IV} / \mathrm{BID}$, meloxicam $0,1 \mathrm{mg} / \mathrm{kg} / \mathrm{IV} / \mathrm{SID}$, dipirona $25 \mathrm{mg} / \mathrm{kg} / \mathrm{IV} / \mathrm{BID}$ e cefazolina $30 \mathrm{mg} / \mathrm{kg} / \mathrm{SC} / \mathrm{BID}$.

No dia seguinte foi realizado o procedimento de herniorrafia inguinal associado a OSH. Como medicação pré-anestésica o animal recebeu dexmedetomidina $5 \mathrm{mc} / \mathrm{kg} / \mathrm{IM}$, midazolan $0,3 \mathrm{mg} / \mathrm{kg} / \mathrm{IM}$ e metadona $0,2 \mathrm{mg} / \mathrm{kg} / \mathrm{IM}$. Em seguida o animal foi induzido com propofol $4 \mathrm{mg} / \mathrm{kg} / \mathrm{IV}$, seguido de intubação orotraqueal e realização de anestesia local via epidural com lidocaína na dose de $0,2 \mathrm{ml} / \mathrm{kg}$. A manutenção anestésica foi realizada com infusão contínua de propofol na dose de $0,2 \mathrm{mg} / \mathrm{kg} / \mathrm{min} / \mathrm{IV}$. Em seguida a paciente foi posicionada em decúbito dorsal e realizada a tricotomia seguida de antissepsia de toda a região abdominal (Figura 2A). A partir de uma incisão envolvendo pele, tecido subcutâneo e musculatura, foi possível a visualização de alças intestinais e útero, no interior da hérnia (Figura 2B). Após o debridamento do anel herniário, o útero e alças intestinais foram delicadamente reposicionados para dentro da cavidade abdominal (Figura 2C), seguido de herniorrafia com sutura de mayo utilizando fio absorvível (Figura 2D) e dermorrafia com fio não absorvível. 


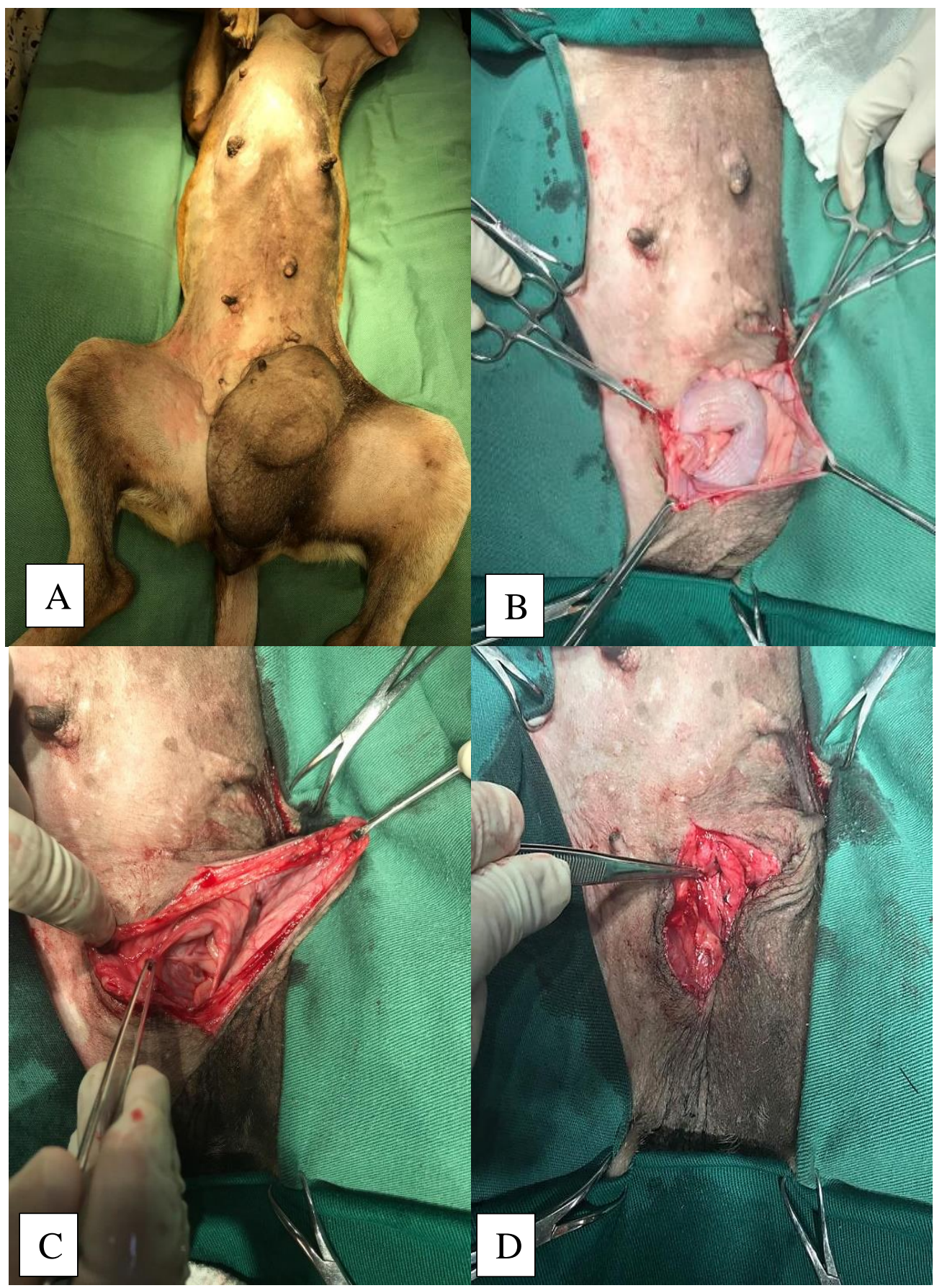

Figura 2. A. Animal em decúbito dorsal evidenciando a hérnia inguinal durante a preparação antecedente aos procedimentos cirúrgicos. B. Estruturas observadas após abertura do saco herniário. C. Reposicionamento de vísceras herniadas para dentro da cavidade abdominal. D. Realização da herniorrafia.

Posteriormente foi realizado a ovariosalpingohisterectomia (OSH) por abordagem pela linha média notando-se o útero distendido e com conteúdo de consistência firme em seu interior (Figura 3A). Instituiu-se então exploração completa do abdômen seguido de celiorrafia (Figura 3B). Após o procedimento foi realizado abertura do mesmo a fim de visualizar o conteúdo, onde foi possível observar secreção escura e fragmentos ósseos resultantes da maceração fetal (Figura 3C e D).

No pós-operatório a paciente permaneceu internada por mais 24 horas, mantendo-se a prescrição de medicamentos. Sendo assim, foi instituído alta médica para a paciente, concomitante a prescrição de cefalexina $30 \mathrm{mg} / \mathrm{kg} / \mathrm{VO} / \mathrm{BID}$ durante 10 dias, meloxican $0,1 \mathrm{mg} / \mathrm{kg} / \mathrm{VO} / \mathrm{SID}$ durante 3 dias, tramadol 4 $\mathrm{mg} / \mathrm{kg} / \mathrm{VO} / \mathrm{BID}$ durante 5 dias e dipirona $25 \mathrm{mg} / \mathrm{kg} / \mathrm{VO} / \mathrm{BID}$ durante 5 dias, assim como, limpeza diária 
da ferida cirúrgica, restrição de exercício e utilização de colar elizabetano. Solicitou-se o retorno em dez dias, para a retirada dos pontos cutâneos. No dia do retorno, a ferida cirúrgica apresentou-se com aspecto de cicatrização e com ausência de secreções.

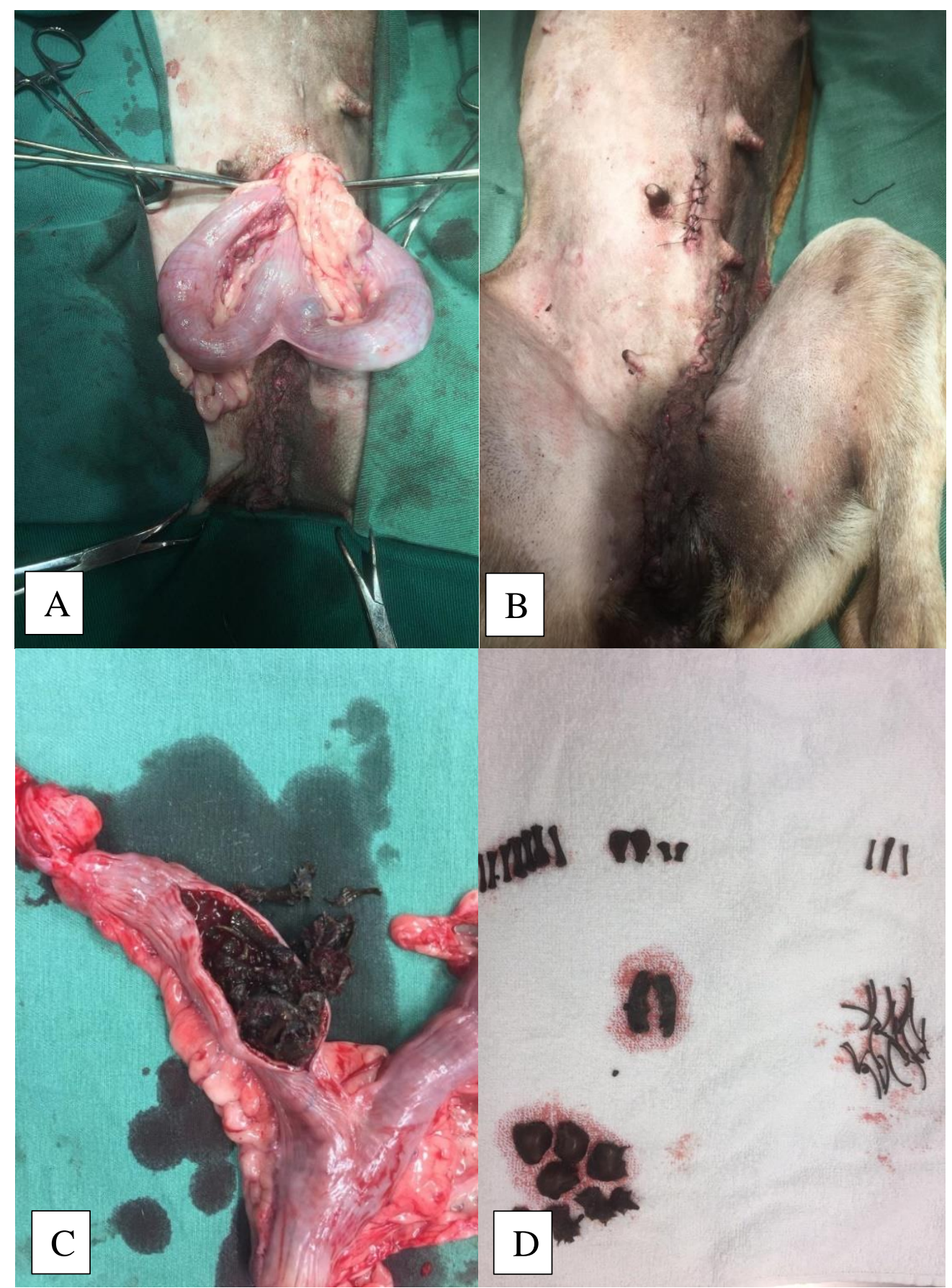

Figura 3. A. útero distendido observado durante o procedimento de ovariosalpingohisterectomia (OSH). B. Procedimento de celiorrafia. C. Presença de fragmentos ósseos em corno uterino aberto após procedimento de OSH. D. Fragmentos ósseos removidos do corno uterino em evidência.

\section{Discussão}

Hérnias inguinais são frequentemente visualizadas em cadelas de meia idade não castradas (Bojrab, 2005). Foster (2013) e Sturion et al. (2013) afirmam que não há predileção racial e que a ocorrência entre outros fatores está relacionada a distúrbios hormonais, como observado no caso relatado, tendo em vista que a paciente apresentava alterações hormonais devido a administração de contraceptivos. Fossum (2014) afirma ser possível que o conteúdo abdominal adentre o subcutâneo devido a um defeito 
no anel inguinal, quando este conteúdo ou parte dele é correspondente ao útero, caracteriza-se o quadro de histerocele. Assim como observado na paciente deste relato.

Ao exame clínico observou-se aumento significativo de volume na região hipogástrica esquerda, originando a hérnia inguinal. Parte do conteúdo presente era o útero, com evidente calcificação fetal, confirmado a partir da radiografia como descrito por (Bellenger, 1980; Cousty et al., 2010; Penaforte Júnior et al., 2017; Ramírez et al., 2015).

O sangramento vaginal apresentado pela paciente deste relato condiz com a literatura. Para Prestes \& Landim-Alvarenga (2017) essa secreção pode ser fluida ou espessa, amarelado, hemorrágico, castanho ou marrom cor de vinho, fétido e eventualmente com tecidos moles e ossos. Neste relato a paciente apresentou maceração fetal, Schlafer (2008) e Tønnessen et al. (2012) afirmam que, as perdas gestacionais podem causar reabsorção embrionária, aborto, mumificação ou maceração fetal e natimortalidade. Ainda neste contexto Tønnessen et al. (2012) concluíram que, o maior índice de morte fetal relaciona-se com o final da gestação, no período perinatal.

O tratamento cirúrgico pela herniorrafia e ovariosalpingohisterectomia (OSH) é eleição para estes casos, sendo a principal forma de resolução para estas patologias, como prescrito por Fossum (2014). Para Loretti et al. (2004) uma das principais causa de maceração fetal é devido a utilização de métodos contraceptivos. Para Toniollo \& Vicente (2003) a utilização indevida destes fármacos gera consequências severas como liquefação e esqueletização. No caso relatado a paciente recebeu contraceptivos por 2 anos seguidos e após o procedimento cirúrgico foi possível visualizar fragmentos ósseos retirados do interior do útero, como afirmado pelos autores.

Os cuidados pós-operatórios pela restrição de exercício, utilização do colar elizabetano e limpeza da ferida cirúrgica, como realizados no caso relatado, foi prescrito por Fossum (2014). A autora afirma ainda que, quando estes cuidados são realizados o prognóstico torna-se favorável aumentando as chances de recuperação do paciente, como observado no caso em questão através do retorno do animal na data marcada.

\section{Conclusão}

A partir do relato apresentado, é evidente a importância do diagnóstico por imagem e do correto tratamento clínico e cirúrgico, que neste caso foi instituído de maneira correta, tendo em vista a recuperação do paciente sem que fosse observadas complicações trans ou pós-operatórias. Evidencia-se também o importante tratamento medicamentoso para a estabilização e recuperação, assim como os cuidados no pós-operatório e os malefícios obtidos através do uso indevido de métodos contraceptivos.

\section{Referências bibliográficas}

Bellenger, C. R. (1980). Perineal hernia in dogs. Australian Veterinary Journal, 56(9):434-438.

Bojrab, M. J. (2005). Técnicas atuais em cirurgia de pequenos animais. São Paulo: Editora Roca.

Booth, N. H. \& McDonaldo, L. E. (1992). Farmacologia e terapêutica em veterinária. Rio de Janeiro, Brasil: Guanabara Koogan.

Costa Neto, J. M., Menezes, V. P., Toríbio, J. M. M. L., Oliveira, É. C. S., Anunciação, M. C., Teixeira, R. G., . . Vieira Júnior, A. S. (2006). Tratamento cirúrgico para correção de hérnia perineal em cão com saculação retal coexistente:(Relato de caso). Revista Brasileira de Saúde e Produção Animal, 7(1):7-19.

Cousty, M., Tricaud, C., Picandet, V. \& Geffroy, O. (2010). Inguinal rupture with herniation of the urinary bladder through the scrotal fascia in a Shetland pony foal. Equine Veterinary Education, 22(1):3-6.

Daleck, C. R., Padilha Filho, J. G., Daleck, C. L. M. \& Costa Neto, J. M. (1992). Reparação de hérnia perineal em cães com peritônio de bovino conservado em glicerina. Ciência Rural, 22(2):179-183.

Fossum, T. W. (2014). Cirurgia de pequenos animais (4 ed. Vol. 1). São Paulo: Elsevier Brasil.

Foster, R. A. (2013). Sistema reprodutor do macho. In J. F. Zachary \& M. D. Mcgavin (Eds.), Bases da patologia veterinária. Rio de Janeiro, Brasil: Elsevier. 
Loretti, A. P., Ilha, M. R. S., Breitsameter, I. \& Faraco, C. S. (2004). Clinical and pathological study of feline mammary fibroadenomatous change associated with depot medroxyprogesterone acetate therapy. Arquivo Brasileiro de Medicina Veterinária e Zootecnia, 56(2):270-274.

Penaforte Júnior, M. A., Aleixo, G. A. S., Maranhão, F. E. C. B. \& Andrade, L. S. S. (2017). Hérnia perineal em cães: revisão de literatura. Medicina Veterinária, 9(1-4):26-35.

Prestes, N. C. \& Landim-Alvarenga, F. C. (2017). Obstetrícia veterinária. Rio de Janeiro, Brasil: Guanabara Koogan.

Ramírez, A., Pastor, N., Durán, M. E., Gutiérrez, A. \& Ezquerra, L. J. (2015). Hernia perineal en el perro, un estudio de prevalencia de 81 casos. Archivos de Medicina Veterinaria, 47(1):71-75.

Schlafer, D. H. (2008). Canine and feline abortion diagnostics. Theriogenology, 70(3):327-331.

Slatter, D. H. (2007). Manual de cirurgia de pequenos animais. São Paulo: Manole.

Sturion, D. J., Sturion, T. T., Sturion, M. A. T., Garcia, B. A., Martins, E. L. \& Moya-Araujo, C. F. (2013). Histerocele inguinal com hematometra em cadela-Relato de Caso. Arquivos de Ciências Veterinárias e Zoologia da UNIPAR, 16(2):165-168.

Toniollo, G. H. \& Vicente, W. R. R. (2003). Manual de obstetrícia veterinária. São Paulo: Varela.

Tønnessen, R., Borge, K. S., Nødtvedt, A. \& Indrebø, A. (2012). Canine perinatal mortality: a cohort study of 224 breeds. Theriogenology, 77(9):1788-1801.

Recebido: 14 de dezembro, 2019.

Aprovado: 19 de janeiro, 2020 .

Publicado: 21 de março, 2020.

Licenciamento: Este artigo é publicado na modalidade Acesso Aberto sob a licença Creative Commons Atribuição 4.0 (CC-BY 4.0), a qual permite uso irrestrito, distribuição, reprodução em qualquer meio, desde que o autor e a fonte sejam devidamente creditados. 